\title{
The Biginelli dihydropyrimidone synthesis using polyphosphate ester as a mild and efficient cyclocondensation/dehydration reagent ${ }^{1}$
}

\author{
Fabio S. Falsone and C. Oliver Kappe* \\ Institute of Chemistry, Organic and Bioorganic Chemistry, Karl-Franzens-University Graz, \\ Heinrichstrasse 28, A-8010 Graz, Austria \\ E-mail: oliver.kappe@uni-graz.at
}

\section{Dedicated to Professor Fritz Sauter on the occasion of his $70^{\text {th }}$ birthday (received 16 Jan 01; accepted 30 Oct 01; published on the web 07 Nov 01)}

\begin{abstract}
4-Aryldihydropyrimidine-2-(1H)-ones $\mathbf{4 a - z}$ were synthesized by one-pot, polyphosphate estermediated Biginelli three-component condensation. The yields of dihydropyrimidones obtained via this novel protocol are significantly higher than those utilizing the conventional ethanol/ $\mathrm{HCl}$ method. The mechanism of the polyphosphate ester based method and the formation of sideproducts are also described.
\end{abstract}

Keywords: Biginelli reaction, cyclocondensation, multicomponent reaction, combinatorial chemistry

\section{Introduction}

In 1893 Italian chemist Pietro Biginelli reported on the acid-catalyzed cyclocondensation reaction of ethyl acetoacetate (1), benzaldehyde (2) and urea (3). ${ }^{2}$ The reaction was carried out simply by heating a mixture of the three components dissolved in ethanol with a catalytic amount of $\mathrm{HCl}$ at reflux temperature. The product of this novel one-pot, three-component synthesis that precipitated on cooling of the reaction mixture was identified correctly by Biginelli as 3,4dihydropyrimidin-2(1H)-one 4 (Scheme 1). ${ }^{2}$

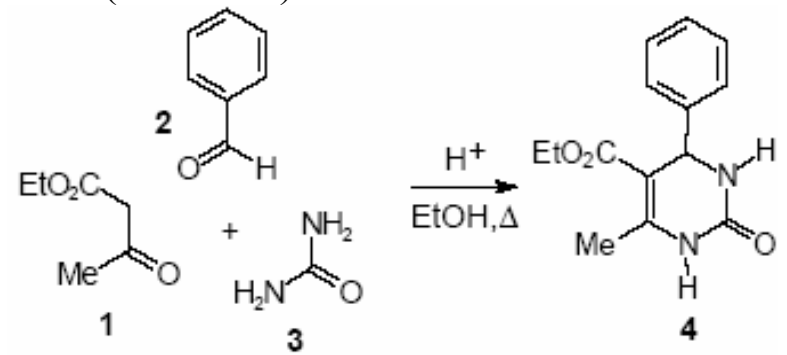

Scheme 1. The Biginelli dihydropyrimidine synthesis. 
The synthetic potential of this new heterocycle synthesis (now known as Biginelli reaction) remained unexplored for quite some time. In the 1970's and 1980's interest slowly increased, and the scope of the original cyclocondensation reaction shown in Scheme 1 was gradually extended by variation of all three building blocks, allowing access to a large number of multifunctionalized dihydropyrimidines of type $4 .^{3}$<smiles>CC(=O)C1=C(C)NC(=O)N(C(N)=O)C1c1cccc([N+](=O)[O-])c1</smiles>

5, SQ 32926<smiles>CC(=O)OC1=C(C)NC(=O)N(C(=O)NN2CCN(c3ccccc3C(N)=O)CC2)C1c1ccc(F)c(F)c1</smiles>

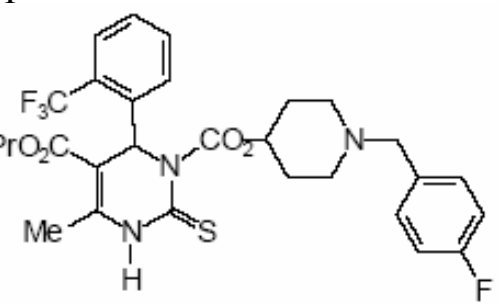

6, SQ 32547<smiles>CC[C@H]1C[C@@H]2CC[C@@H]3C(C(=O)OCCCCC4NC(=N)N5CCCC5=C4C(=O)OCCNC(N)=[NH2+])=C(C)NC(N1)N23</smiles>

Figure 1. Examples of biologically active DHPMs

Since the late 1980's a tremendous increase in activity has again occurred, as evidenced by the growing number of publications and patents on the subject. ${ }^{3,4}$ This is mainly due to the fact that the multifunctionalized dihydropyrimidine scaffold 4 (DHPMs, "Biginelli compounds") represents a heterocyclic system of remarkable pharmacological efficiency. In the past decades, a broad range of biological effects, including antiviral, antitumor, antibacterial, and antiinflammatory activities has been ascribed to these partly reduced pyrimidine derivatives. ${ }^{5}$ More recently, appropriately functionalized DHPMs have emerged as e.g. orally active antihypertensive agents $5,6^{6-8}$ or $\alpha_{1}$ a adrenoceptor-selective antagonists (7). ${ }^{9}$ A very recent highlight in this context has been the identification of the structurally rather simple DHPM monastrol (8) as a mitotic kinesin Eg5 motor protein inhibitor and potential new lead for the development of anticancer drugs. ${ }^{10}$ Apart from synthetic DHPM derivatives, several marine natural products with interesting biological activities containing the dihydropyrimidine-5carboxylate core have recently been isolated. ${ }^{11}$ Most notably among these are the batzelladine 
alkaloids A and B (e.g. 9) which inhibit the binding of HIV envelope protein gp-120 to human CD4 cells and, therefore, are potential new leads for AIDS therapy. ${ }^{12}$

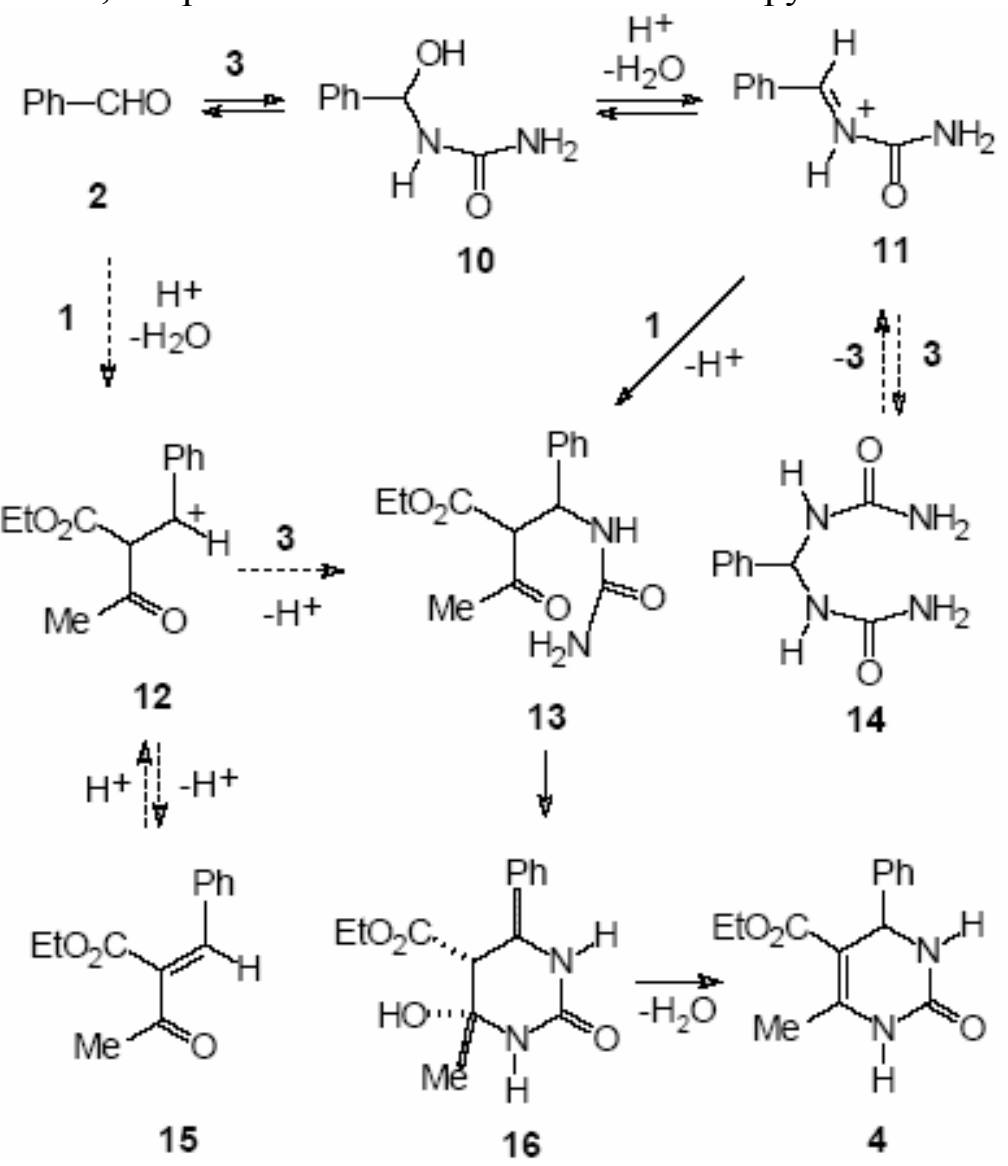

Scheme 2. The mechanism of the Biginelli reaction.

Although the most straightforward protocol to synthesize DHPMs 4 is the one-pot acidcatalyzed Biginelli condensation shown above (Scheme 1), this protocol - using ethanol and catalytic amounts of $\mathrm{HCl}$ - often provides only low to moderate yields of the desired target molecules of type 4, in particular, when substituted aromatic aldehydes or thioureas are employed. ${ }^{3,13-15}$ This has led to the recent disclosure of several improved reaction protocols for the synthesis of DHPMs, either by modification of the classical one-pot Biginelli approach itself, ${ }^{13}$ or by the development of novel, but more complex multistep strategies. ${ }^{14}$ In addition, several combinatorial approaches towards DHPMs 4 have been advanced,15 using e.g. solid phase, ${ }^{15 a, b}$ or fluorous phase ${ }^{15 c, d}$ reaction conditions.

We have investigated the mechanism of the Biginelli reaction in 1997 using ${ }^{1} \mathrm{H} /{ }^{13} \mathrm{C} \mathrm{NMR}$ spectroscopy and trapping experiments, and have established that the key step in this sequence involves the acid-catalyzed formation of an $N$-acyliminium ion intermediate of type $\mathbf{1 1}$ from the aldehyde $\mathbf{2}$ and urea $\mathbf{3}$ precursors. ${ }^{16}$ Interception of the iminium ion $\mathbf{1 1}$ by ethyl acetoacetate $\mathbf{1}$, presumably through its enol tautomer, produces an open chain ureide $\mathbf{1 3}$ which subsequently cyclizes to hexahydropyrimidine 16. Acid-catalyzed elimination of water from 16 ultimately 
leads to the final DHPM product 4. The reaction mechansim can therefore be classified as an $\alpha$ amidoalkylation, or more specifically as an $\alpha$-ureidoalkylation. ${ }^{17}$

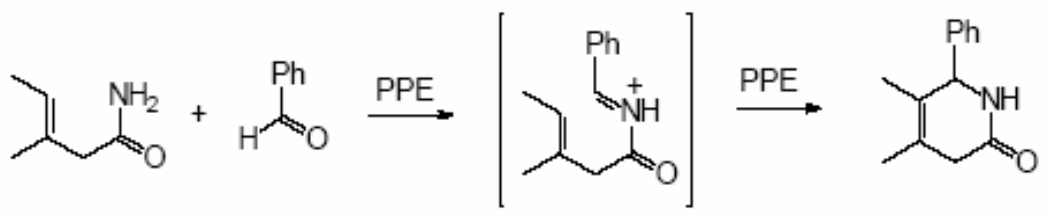

\section{Scheme 3}

In order to promote conditions that would favor the formation and interception of such an iminium ion intermediate (i.e. 11) in the Biginelli reaction - thereby minimizing side reactions we have now investigated a variety of reaction conditions more specifically employed in $\mathrm{N}$ acyliminium ion based amidoalkylations. ${ }^{18}$ One of the most efficient reagents tested proved to be polyphosphate ester (PPE), which has been demonstrated to mediate acyliminium ion-based condensations in the past such as the somewhat related dihydropyridone formations shown in Scheme 3. ${ }^{19,20}$ Here we report in full detail the development of improved conditions for performing Biginelli condensations, using PPE as mild cyclization reagent. ${ }^{21}$

\section{Results and Discussion}

Polyphosphate ester (PPE) is one of the phosphate-based reagents that are related to polyphosphoric acid (PPA). It differs from PPA in that it is aprotic and soluble in organic media. It is often compared to the related polyphosphoric acid trimethylsilyl ester (PPSE). In general, the advantages of PPE include its solubility in organic solvents, the mild conditions under which it is used, and its relatively nonhazardous, nonnoxious nature. ${ }^{19}$ Although the reagent is not commercially available it is readiliy prepared by treatment of phosphorous oxide with anhydrous ether (see Experimental). As reagent, PPE has been used successfully in Bischler-Napieralski reactions, Friedel-Crafts acylations, Beckmann rearrangements, and in the conversion of carboxylic acids to esters, thiol esters, amides, and nitriles. ${ }^{19}$

Our initial attempts focused on the use of neat PPE as reaction mediator (i.e. solvent) as described by Marson for similar cyclizations. ${ }^{20}$ Although the yields of crude dihydro-pyrimidone 4a were respectable (ca. $80 \%$ ), the solubility of urea in cold PPE and side-reactions leading to unwanted by-products (see below) initially created a problem. In addition, for complete conversion reaction times of $24 \mathrm{~h}$ at $40{ }^{\circ} \mathrm{C}$ were necessary. This method was further complicated by the work-up procedure: adding a large amount of water or ice to the reaction mixture resulted not only in the precipitation of the desired product, but also precipitated by-products such as the Knoevenagel product 15 (see Scheme 2) ${ }^{16}$ and the 6-styryl-derivative 18 (see below). Although we have recently shown that by using a microwave-assisted protocol these problems can be overcome and neat PPE can still be used effectively (in particular for the parallel and small-scale synthesis of DHPM libraries); ${ }^{22}$ we felt that using a co-solvent could also eliminate some of the 
problems with neat PPE and make the protocol more amenable to a larger-scale preparation of DHPMs.

After some experimentation with respect to the molar ratio of reactants, the reaction temperature/time, and the nature of the co-solvent we have found a set of conditions that consistently produced good to excellent yields of dihydropyrimidones 4a-z. These conditions utilized a 1:1:1.5 ratio of $\beta$-ketoester, aldehyde, and urea derivative in a one-pot condensation employing anhydrous refluxing THF as solvent which had previously been employed successfully in the Biginelli condensation.15a,d The presence of PPE as a reaction mediator (150 $\mathrm{mg}$ of per mmol of reactants) provided the highest yields as discovered by a simple optimization study (Table 1).

Table 1. Influence of reaction time and amount of PPE on the yield of DHPM 4a

\begin{tabular}{cccc}
\hline Reaction Time (h) / PPE (mg) & \multicolumn{3}{c}{ Yield (\%) } \\
\hline & $\mathbf{5}$ & $\mathbf{1 0}$ & $\mathbf{1 5}$ \\
50 & 52 & 75 & 77 \\
100 & 63 & 77 & 87 \\
150 & 69 & 75 & 94 \\
\hline
\end{tabular}

After the reaction was completed the mixture was simply poured onto a limited amount of crushed ice, whereupon the solid dihydropyrimidines 4a-z (Table 2) precipitated from the reaction mixture, but minor amounts of by-products $(<5 \%)$ remained in solution. Based on ${ }^{1} \mathrm{H}$ NMR measurements $(200 \mathrm{MHz})$ these products had at least 98\% purity. For comparison purposes, the traditional Biginelli conditions $(\mathrm{EtOH} / \mathrm{HCl} \text {, reflux })^{3}$ also provided dihydropyrimidines 4a-z (yields are not shown). Where yields were not available from the literature the corresponding dihydropyrimidines were synthesized according to the classical Biginelli conditions described by Folkers et al. ${ }^{23}$ For all cases th investigated the PPE/THF method produced significantly higher yields $(30-40 \%$ on average) than the classical Biginelli method. Apart from its simplicity, one important feature of this novel protocol is the ability to tolerate variations in all three components. Beside urea, $N$-substituted ureas can be used without

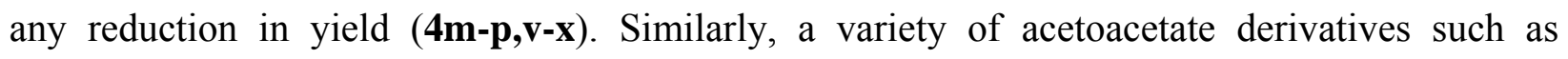
methyl- (q,r), isopropyl- (s,t), tert.-butyl- $(\mathbf{u})$, benzyl- $(\mathbf{v}, \mathbf{w})$, and allyl acetoacetate $(\mathbf{x})$ can be employed. Most importantly, the aromatic aldehyde can be varied to a large extent and many of the pharmacologically relevant ${ }^{5}$ substitution patterns on the 4-aryl ring (e.g. 4e-k) can be introduced with high efficiency. The yields of these dihydropyrimidines using the one-pot PPE/THF protocol compare very favorably with the overall yields achievable utilizing the currently best and most frequently used multi-step method ( 3 steps) disclosed by Atwal ${ }^{14 a}$ (i.e. $4 \mathbf{f}$ : 80\%, 4i: 63\%, 4j: 69\%). Even for aliphatic aldehydes (i.e. acetaldehyde) which normally show extremely poor yields in the Biginelli reaction ${ }^{3,23,24}$ a $53 \%$ yield of the corresponding pyrimidine $\mathbf{4 z}$ was obtained. 
Table 2. Polyphosphate Ester-Mediated Synthesis of DHPMs 4

\begin{tabular}{|c|c|c|c|c|}
\hline DHPM & $\mathrm{R}_{1}$ & $\mathrm{R}$ & $\mathrm{R} 2$ & Yield (\%) \\
\hline $4 a$ & $\mathrm{C}_{6} \mathrm{H}_{5}$ & Et & $\mathrm{H}$ & 94 \\
\hline $4 b$ & $3,4-(\mathrm{MeO})_{2}-\mathrm{C}_{6} \mathrm{H}_{3}$ & Et & $\mathrm{H}$ & 75 \\
\hline 4c & 2-( $(\mathrm{MeO})-\mathrm{C}_{6} \mathrm{H}_{4}$ & Et & $\mathrm{H}$ & 83 \\
\hline 4d & $4-(\mathrm{Me})-\mathrm{C}_{6} \mathrm{H}_{4}$ & $\mathrm{Et}$ & $\mathrm{H}$ & 86 \\
\hline $4 e$ & $4-\left(\mathrm{NO}_{2}\right)-\mathrm{C}_{6} \mathrm{H}_{4}$ & Et & $\mathrm{H}$ & 77 \\
\hline 4f & $3-\left(\mathrm{NO}_{2}\right)-\mathrm{C}_{6} \mathrm{H}_{4}$ & Et & $\mathrm{H}$ & 87 \\
\hline $4 g$ & $2-\left(\mathrm{NO}_{2}\right)-\mathrm{C}_{6} \mathrm{H}_{4}$ & Et & $\mathrm{H}$ & 84 \\
\hline $4 h$ & $2-(\mathrm{Cl})-\mathrm{C}_{6} \mathrm{H}_{4}$ & Et & $\mathrm{H}$ & 83 \\
\hline $4 i$ & $2,3-(\mathrm{Cl})_{2}-\mathrm{C}_{6} \mathrm{H}_{3}$ & Et & $\mathrm{H}$ & 79 \\
\hline $4 j$ & $2-\left(\mathrm{CF}_{3}\right)-\mathrm{C}_{6} \mathrm{H}_{4}$ & Et & $\mathrm{H}$ & 68 \\
\hline $4 \mathbf{k}$ & $3,4-(\mathrm{F})_{2}-\mathrm{C}_{6} \mathrm{H}_{3}$ & Et & $\mathrm{H}$ & 84 \\
\hline 41 & 2-Thienyl & Et & $\mathrm{H}$ & 86 \\
\hline $4 m$ & $\mathrm{C}_{6} \mathrm{H}_{5}$ & Et & $\mathrm{Me}$ & 95 \\
\hline $4 n$ & $4-\left(\mathrm{NO}_{2}\right)-\mathrm{C}_{6} \mathrm{H}_{4}$ & Et & $\mathrm{Me}$ & 99 \\
\hline 40 & $3-\left(\mathrm{NO}_{2}\right)-\mathrm{C}_{6} \mathrm{H}_{4}$ & Et & $\mathrm{Me}$ & 86 \\
\hline $4 p$ & $\mathrm{C}_{6} \mathrm{H}_{5}$ & Et & $\mathrm{CH}_{2} \mathrm{Ph}$ & 91 \\
\hline $4 q$ & 2-(Me)- $\mathrm{C}_{6} \mathrm{H}_{4}$ & $\mathrm{Me}$ & $\mathrm{H}$ & 88 \\
\hline $4 r$ & $4-\left(\mathrm{NO}_{2}\right)-\mathrm{C}_{6} \mathrm{H}_{4}$ & $\mathrm{Me}$ & $\mathrm{H}$ & 84 \\
\hline $4 s$ & $\mathrm{C}_{6} \mathrm{H}_{5}$ & $i-\operatorname{Pr}$ & $\mathrm{H}$ & 84 \\
\hline $4 t$ & $3-\left(\mathrm{NO}_{2}\right)-\mathrm{C}_{6} \mathrm{H}_{4}$ & $i-\operatorname{Pr}$ & $\mathrm{H}$ & 88 \\
\hline $4 u$ & $\mathrm{C}_{6} \mathrm{H}_{5}$ & $t-\mathrm{Bu}$ & $\mathrm{H}$ & 77 \\
\hline $4 v$ & $\mathrm{C}_{6} \mathrm{H}_{5}$ & $\mathrm{CH}_{2} \mathrm{Ph}$ & $\mathrm{Me}$ & 93 \\
\hline $4 w$ & $2,3-(\mathrm{Cl})_{2}-\mathrm{C}_{6} \mathrm{H}_{3}$ & $\mathrm{CH}_{2} \mathrm{Ph}$ & $\mathrm{Me}$ & 93 \\
\hline $4 x$ & $3-\left(\mathrm{NO}_{2}\right)-\mathrm{C}_{6} \mathrm{H}_{4}$ & Allyl & $\mathrm{Me}$ & 85 \\
\hline $4 y$ a & $\mathrm{C}_{6} \mathrm{H}_{5}-\mathrm{C} \equiv \mathrm{C}$ & $\mathrm{Et}$ & $\mathrm{H}$ & 92 \\
\hline $4 z_{b}$ & $\mathrm{Me}$ & $\mathrm{Et}$ & $\mathrm{H}$ & $53 \mathrm{c}$ \\
\hline
\end{tabular}

${ }^{a} 1.5$ equiv of aldehyde used. ${ }^{b}$ Molar ratio 1:1.5:1.5 of urea, acetaldehyde, and ethyl acetoacetate (sealed tube, $50{ }^{\circ} \mathrm{C}$ ). ${ }^{\mathrm{c}}$ After chromatographic work-up.

The success of the PPE/THF method may be the result of specific interaction of PPE with the $\mathrm{N}$-acyliminium ion intermediates of type 11, possibly involving a stabilized enol phosphate intermediate of type $\mathbf{1 7}^{20}$ (Figure 2). The dehydrating properties of $\mathrm{PPE}^{19}$ in combination with the aprotic THF medium may further drive the reaction along the desired pathway and minimize side reactions that frequently occur when employing the traditional Biginelli protocol. Despite longer 
reaction times the PPE/THF method appears to be somewhat gentler than the classical protic solvent conditions utilizing mineral acid catalysis. The only limitation of the current protocol concerns the use of acetoacetamides. Here, the Biginelli condensation gives only low yields of the corresponding DHPMs, presumably due to competitive interaction of PPE with the amide moiety in acetoacetamides. With thioureas the PPE protocol gives similar yields as obtained using the conventional EtOH/HCl method. A corresponding solvent-free, "dry media" synthesis gives much better results. ${ }^{23,25}$

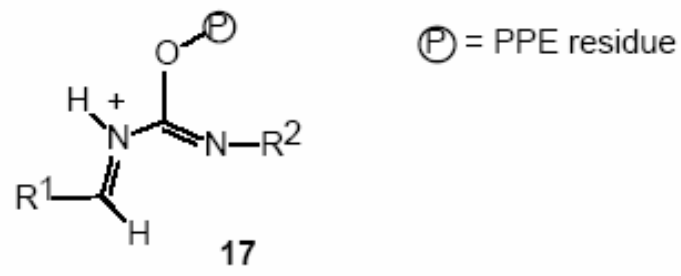

Figure 2. Structure of stabilized $\mathrm{N}$-acyliminium ion intermediate.

Finally, we wish to report here on the formation of an hitherto unobserved type of by-product in the Biginelli reaction. Employing the neat PPE reaction conditions $\left(40^{\circ} \mathrm{C}, 24 \mathrm{~h}\right)$ the formation of a by-product was clearly observed by TLC and NMR analysis of the crude reaction mixture (5-10\%). The structure of this product, i.e. the 6-styryl-derivative 18, could be confirmed by independent synthesis and comparison with the authentic material (this work). Interestingly, 18 was not formed by reaction of $4 \mathbf{a}$ with benzaldehyde under the PPE conditions as confirmed by an independent experiment. In order to force this condensation process, much more drastic reaction conditions had to be applied (refluxing xylene, $p$-toluenesulfonic acid; Scheme 4). ${ }^{26}$ Therefore, the formation of $\mathbf{1 8}$ can be rationalized only by reaction of benzaldehyde with one of the early intermediates in the Biginelli condensation process (i.e. 13, Scheme 2), although no attempts were made to clarify this point.

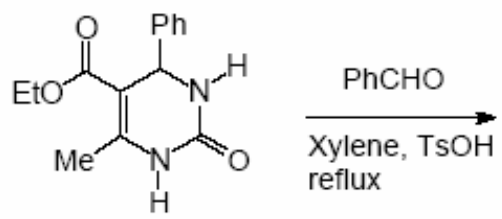

$4 \mathbf{a}$

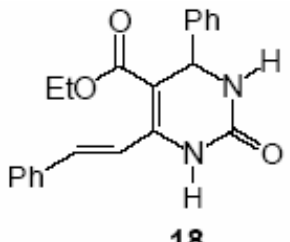

18

\section{Scheme 4}

\section{Conclusions}

In summary, we have disclosed a new and simple modification of the Biginelli dihydropyrimidine synthesis. Through the use of readily available polyphosphate ester (PPE) as reaction mediator and THF as solvent the yields in the one-pot Biginelli protocol can be increased on average by $30-40 \%$ as compared with the traditional methods. This one-step synthesis of dihydropyrimidines therefore is superior in simplicity and yield compared to alternative multistep strategies that have been reported. The use of PPE in related cyclization reactions is currently underway in our laboratories and will be reported in due course. 


\section{Experimental Section}

General Procedures. Melting points were determined on a Gallenkamp melting point apparatus Mod. MFB-595. ${ }^{1} \mathrm{H}$ NMR spectra were obtained on a Varian XL-200 Gemini instrument (200 MHz). IR spectra were recorded on a Perkin-Elmer 298 spectrophotometer. Elemental analyses were obtained on a Fisons Mod. EA 1108 elemental analyzer. Reactions were monitored by thin layer chromatography on $0.2 \mathrm{~mm}$ silica gel F-252 (Merck) plates. All ß-keto esters 1, aldehydes 2, and urea derivatives 3 were obtained from Aldrich Chemical Co. and used without further purification, with the exception of benzaldehyde, which was distilled in vacuo prior to use. THF was anhydrous grade (Fluka 87371, stored over molecular sieves). All solid components were employed as grained powders.

Polyphosphate ester (modified according to ref.19): $\mathrm{P}_{2} \mathrm{O}_{5}(150 \mathrm{~g})$ was added rapidly with intense stirring to a solution of anhydrous ether $(300 \mathrm{~mL})$ and alcohol-free chloroform $(150 \mathrm{~mL})$. The reaction was heated under reflux in a dry argon atmosphere for $3 \mathrm{~d}$ upon which $\mathrm{P}_{2} \mathrm{O}_{5}$ gradually dissolved. The resulting clear solution was concentrated to a colorless or light-brown syrup in a rotary evaporator; residual traces of solvent were removed by heating the syrup for $24 \mathrm{~h}$ at $40{ }^{\circ} \mathrm{C}$ in vacuo (15 mbar).

\section{General procedure for the PPE-mediated synthesis of dihydropyrimidones 4a-z}

A mixture of the appropriate aldehyde $(2.00 \mathrm{mmol})$, acetoacetate $(2.00 \mathrm{mmol})$, urea $(3.00 \mathrm{mmol})$, and THF $(4 \mathrm{~mL})$ containing PPE $(300 \mathrm{mg})$ was heated under reflux and stirring for $15 \mathrm{~h}$. After cooling the reaction mixture was poured onto crushed ice $(10 \mathrm{~g})$. Stirring was continued for several hours; the solid products were filtered, washed with ice water and subsequently dried. Analytical samples were obtained by recrystallization from appropriate solvents. ${ }^{1} \mathrm{H}$ NMR measurements of these products showed no trace of impurities. All new compounds were characterized by NMR, IR and elemental analysis, or by comparison with authentic samples.

Ethyl 6-methyl-2-oxo-4-phenyl-1,2,3,4-tetrahydropyrimidine-5-carboxylate (4a). Yield $489 \mathrm{mg}(94 \%)$; mp 206-207 ${ }^{\circ} \mathrm{C}$ (ethanol) (lit. ${ }^{23} \mathrm{mp} 202.4^{\circ} \mathrm{C}$ ). IR (KBr): $\left[\mathrm{cm}^{-1}\right] 3240,3110,1725$, 1700, 1645; ' ${ }^{\mathrm{H}}$ NMR (DMSO- $d_{6}$ ): $\delta 1.12$ (t, $J=7.5 \mathrm{~Hz}, 3 \mathrm{H}$ ), 2.28 (s, 3H), 4.03 (q, $J=7.5 \mathrm{~Hz}$, $2 \mathrm{H}), 5.17(\mathrm{~d}, J=3.0 \mathrm{~Hz}, 1 \mathrm{H}), 7.22-7.41(\mathrm{~m}, 5 \mathrm{H}), 7.78(\mathrm{br} \mathrm{s}, 1 \mathrm{H}), 9.22(\mathrm{br} \mathrm{s}, 1 \mathrm{H})$.

Ethyl 4-(3,4-dimethoxyphenyl)-6-methyl-2-oxo-1,2,3,4-tetrahydropyrimidine-5-carboxylate (4b). Yield $480 \mathrm{mg}$ (75\%), mp $178{ }^{\circ} \mathrm{C}$ (ethanol) (lit. ${ }^{23} 178-178.5{ }^{\circ} \mathrm{C}$ ). IR (KBr): $\left[\mathrm{cm}^{-1}\right]$ 3240, 3100, 1720, 1710, 1650; ${ }^{1} \mathrm{H}$ NMR (DMSO- $d_{6}$ ): $\delta 1.15$ (t, $\left.J=7.5 \mathrm{~Hz}, 3 \mathrm{H}\right), 2.28$ (s, 3H), 3.73 (s, $6 \mathrm{H}), 4.12$ (q, $J=7.5 \mathrm{~Hz}, 2 \mathrm{H}), 5.11(\mathrm{~d}, J=3.0 \mathrm{~Hz}, 1 \mathrm{H}), 6.70-7.95$ (m, 3H), 7.69 (brs, $1 \mathrm{H}), 9.17$ (brs, $1 \mathrm{H})$.

Ethyl 6-methyl-4-(2-methoxyphenyl)-2-oxo-1,2,3,4-tetrahydropyrimidine-5-carboxylate (4c). Yield $435 \mathrm{mg}(83 \%), \mathrm{mp} 262{ }^{\circ} \mathrm{C}$ (ethanol). IR (KBr): $\left[\mathrm{cm}^{-1}\right]$ 3250, 3100, 1730, 1700, 1680; ${ }^{1} \mathrm{H}$ NMR (DMSO- $d_{6}$ ): $\delta 1.08$ (t, $J=7.5 \mathrm{~Hz}, 3 \mathrm{H}$ ), 2.30 (s, 1H), 3.80 (s, 3H, OMe), 3.93 (q, $J=7.5$ $\mathrm{Hz}, 2 \mathrm{H}), 5.50(\mathrm{~d}, J=3.0 \mathrm{~Hz}, 1 \mathrm{H}), 6.82-7.09(\mathrm{~m}, 3 \mathrm{H}), 7.14-7.21(\mathrm{~m}, 2 \mathrm{H}), 9.10$ (brs, 1H); Anal. calcd. for $\mathrm{C}_{15} \mathrm{H}_{18} \mathrm{~N}_{2} \mathrm{O}_{4}$ (290.32): C, 62.06; H, 6.25; N, 9.65. Found: C, 62.19; H, 6.34; N, 9.48. 
Ethyl 6-methyl-4-(4-methylphenyl)-2-oxo-1,2,3,4-tetrahydropyrimidine-5-carboxylate (4d). Yield $471 \mathrm{mg}(86 \%), \mathrm{mp} 212{ }^{\circ} \mathrm{C}$ (ethanol). IR (KBr): [ $\left.\mathrm{cm}^{-1}\right]$ 3220, 3100, 1720sh, 1700; ${ }^{1} \mathrm{H}$ NMR $\left(\mathrm{DMSO}-d_{6}\right): \delta 1.12(\mathrm{t}, J=7.5 \mathrm{~Hz}, 3 \mathrm{H}), 2.28,2.30(2 \mathrm{~s}, 6 \mathrm{H}), 4.00(\mathrm{q}, J=7.5 \mathrm{~Hz}, 2 \mathrm{H}), 5.11(\mathrm{~d}, J=$ $3.0 \mathrm{~Hz}, 1 \mathrm{H}), 7.12$ (s, 4H), 7.70 (br s, 1H), 9.19 (brs, 1H).

Ethyl 6-methyl-4-(4-nitrophenyl)-2-oxo-1,2,3,4-tetrahydropyrimidine-5-carboxylate

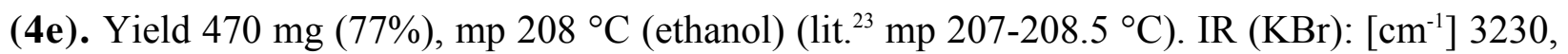
3120, 1730, 1710, 1650; ${ }^{1} \mathrm{H}$ NMR (DMSO-d $): \delta 1.11(\mathrm{t}, J=7.5 \mathrm{~Hz}, 3 \mathrm{H}), 2.29(\mathrm{~s}, 3 \mathrm{H}), 4.00(\mathrm{q}, J$ $=7.5 \mathrm{~Hz}, 2 \mathrm{H}), 5.29(\mathrm{~d}, J=3.0 \mathrm{~Hz}, 1 \mathrm{H}), 7.51(\mathrm{~d}, J=10 \mathrm{~Hz}, 2 \mathrm{H}), 7.91(\mathrm{br} \mathrm{s}, 1 \mathrm{H}), 8.23(\mathrm{~d}, J=$ $10.0 \mathrm{~Hz}, 2 \mathrm{H}), 9.37$ (br s, 1H).

Ethyl 6-methyl-4-(3-nitrophenyl)-2-oxo-1,2,3,4-tetrahydropyrimidine-5-carboxylate (4f).

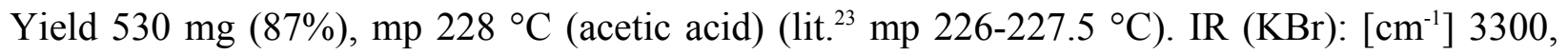
3120, 1710, 1690, 1630; ${ }^{1} \mathrm{H}$ NMR (DMSO-d $): \delta 1.11(\mathrm{t}, J=7.5 \mathrm{~Hz}, 3 \mathrm{H}), 2.29(\mathrm{~s}, 3 \mathrm{H}), 4.02(\mathrm{q}, J$ $=7.5 \mathrm{~Hz}, 2 \mathrm{H}), 5.31(\mathrm{~d}, J=3.0 \mathrm{~Hz}, 1 \mathrm{H}), 7.65-7.75(\mathrm{~m}, 2 \mathrm{H}), 7.95$ (br s, $1 \mathrm{H}), 8.09-8.20(\mathrm{~m}, 2 \mathrm{H})$, 9.34 (br s, $1 \mathrm{H})$.

Ethyl 6-methyl-4-(2-nitrophenyl)-2-oxo-1,2,3,4-tetrahydropyrimidine-5-carboxylate (4g). Yield $512 \mathrm{mg}(84 \%), \mathrm{mp} 220{ }^{\circ} \mathrm{C}$ (acetic acid/methanol) (lit. $\left.{ }^{28} 218-220{ }^{\circ} \mathrm{C}\right)$. IR (KBr): $\left[\mathrm{cm}^{-1}\right]$ 3240, 3100, 1710, 1650; ${ }^{1} \mathrm{H}$ NMR (DMSO-d 6 ): $\delta 0.94$ (t, $\left.J=7.5 \mathrm{~Hz}, 3 \mathrm{H}\right), 2.30(\mathrm{~s}, 1 \mathrm{H}), 3.88$ (q, $J$ $=7.5 \mathrm{~Hz}, 2 \mathrm{H}), 5.81(\mathrm{~d}, \mathrm{~J}=3.0 \mathrm{~Hz}, 1 \mathrm{H}), 7.49-7.98(\mathrm{~m}, 5 \mathrm{H}), 9.39$ (br s, $1 \mathrm{H})$.

Ethyl 4-(2-chlorophenyl)-6-methyl-2-oxo-1,2,3,4-tetrahydropyrimidine-5-carboxylate (4h). Yield $488 \mathrm{mg}(83 \%), \mathrm{mp} 215-218{ }^{\circ} \mathrm{C}$ (ethanol) $\left(\mathrm{lit}^{29} 214{ }^{\circ} \mathrm{C}\right) . \mathrm{IR}(\mathrm{KBr}):\left[\mathrm{cm}^{-1}\right]$ 3360, 3220, 3100, 1690, 1640; ${ }^{1} \mathrm{H}$ NMR (DMSO- $\left.d_{6}\right) \delta 1.08(\mathrm{t}, J=7.5 \mathrm{~Hz}, 3 \mathrm{H}), 2.32(\mathrm{~s}, 3 \mathrm{H}), 3.91(\mathrm{q}, J=7.5$ $\mathrm{Hz}, 2 \mathrm{H}), 5.67$ (d, $J=2.5 \mathrm{~Hz}, 1 \mathrm{H}), 7.22-7.46$ (m, 4H), 7.72 (br s, 1H), 9.30 (br s, 1H).

Ethyl 4-(2,3-dichlorophenyl)-6-methyl-2-oxo-1,2,3,4-tetrahydropyrimidine-5-carboxylate (4i). Yield $520 \mathrm{mg}(79 \%), \mathrm{mp} 244-246{ }^{\circ} \mathrm{C}$ (methanol) (lit. $\left.{ }^{14 a} 212-214{ }^{\circ} \mathrm{C}\right) . \mathrm{IR}(\mathrm{KBr}):\left[\mathrm{cm}^{-1}\right] 3360$, 3100, 1700, 1690sh, 1640; ${ }^{1} \mathrm{H}$ NMR (DMSO-d $)^{\text {) }} \delta 0.97$ (t, $\left.J=7.5 \mathrm{~Hz}, 3 \mathrm{H}\right), 2.31$ (s, 3H), 3.89 (q, $J=7.5 \mathrm{~Hz}, 2 \mathrm{H}), 5.69$ (br s, 1H), 7.25-7.43 (m, 2H), 7.50-7.61 (m, 1H), 7.80 (br s, 1H), 9.32 (br s, $1 \mathrm{H})$.

Ethyl 6-methyl-2-oxo-4-(2-trifluoromethylphenyl)-1,2,3,4-tetrahydropyrimidine-5carboxylate (4j). Yield $446 \mathrm{mg}(68 \%), \mathrm{mp} 202-203{ }^{\circ} \mathrm{C}$ (ethanol) (lit. $\left.{ }^{14 \mathrm{a}} 198-200{ }^{\circ} \mathrm{C}\right) . \mathrm{IR}(\mathrm{KBr})$ : $\left[\mathrm{cm}^{-1}\right] 3230,3100,1700,1640 ;{ }^{1} \mathrm{H}$ NMR $\left(\mathrm{CDCl}_{3}\right) \delta 0.97(\mathrm{t}, J=7.5 \mathrm{~Hz}, 3 \mathrm{H}), 2.45(\mathrm{~s}, 3 \mathrm{H}), 3.97$ (q, $J=7.5 \mathrm{~Hz}, 2 \mathrm{H}), 5.37$ (br s, $1 \mathrm{H}), 5.82$ (br s, 1H), 7.32-7.70 (m, 4H), 8.46 (br s, 1H).

Ethyl 4-(3,4-difluorophenyl)-6-methyl-2-oxo-1,2,3,4-tetrahydropyrimidine-5-carboxylate (4k). Yield $497 \mathrm{mg}(84 \%)$, mp 188-189 ${ }^{\circ} \mathrm{C}$ (ethanol) (lit. $\left.{ }^{13 \mathrm{c}} 185-186{ }^{\circ} \mathrm{C}\right) . \mathrm{IR}(\mathrm{KBr}):\left[\mathrm{cm}^{-1}\right] 3320$, 1710, 1680, 1660, 1640; ${ }^{1} \mathrm{H}$ NMR (DMSO-d 6 ) $\delta 1.11(\mathrm{t}, J=7.5 \mathrm{~Hz}, 3 \mathrm{H}), 2.29(\mathrm{~s}, 3 \mathrm{H}), 4.02(\mathrm{q}, J$ $=7.5 \mathrm{~Hz}, 2 \mathrm{H}), 5.17(\mathrm{~d}, J=3.0 \mathrm{~Hz}, 1 \mathrm{H}), 7.05-7.50(\mathrm{~m}, 3 \mathrm{H}), 7.80$ (br s, $1 \mathrm{H}), 9.28$ (br s, $1 \mathrm{H})$.

Ethyl 6-methyl-2-oxo-4-(2-thienyl)-1,2,3,4-tetrahydropyrimidine-5-carboxylate (4l) Yield $457 \mathrm{mg}(86 \%), \mathrm{mp} 220^{\circ} \mathrm{C}$ (ethanol). IR (KBr): $\left[\mathrm{cm}^{-1}\right]$ 3340, 3240, 3120, 1700, 1650, 1630 sh; ${ }^{1} \mathrm{H}-\mathrm{NMR}\left(\mathrm{DMSO}-d_{6}\right): \delta 1.19(\mathrm{t}, J=7.5 \mathrm{~Hz}, 3 \mathrm{H}), 2.28(\mathrm{~s}, 3 \mathrm{H}), 4.10(\mathrm{q}, J=7.5 \mathrm{~Hz}, 2 \mathrm{H}), 5.43$ $(\mathrm{d}, J=3.0 \mathrm{~Hz}, 1 \mathrm{H}), 6.89-7.11(\mathrm{~m}, 2 \mathrm{H}), 7.33-7.42(\mathrm{~d}, J=6.0 \mathrm{~Hz}, 1 \mathrm{H}), 7.92$ (br s, 1H), 9.33 (br s, $1 \mathrm{H}$ ); Anal calcd. for $\mathrm{C}_{12} \mathrm{H}_{14} \mathrm{~N}_{2} \mathrm{O}_{3} \mathrm{~S}$ (266.32): C, 54.12, H, 5.30; N, 10.52. Found: C, 54.23; H, 5.34; N, 10.37. 
Ethyl 1,6-Dimethyl-2-oxo-4-phenyl-1,2,3,4-tetrahydropyrimidine-5-carboxylate (4m). Yield $520 \mathrm{mg}$ (95\%), mp $180{ }^{\circ} \mathrm{C}$ (ethanol) (lit. $\left.{ }^{30} 176-178{ }^{\circ} \mathrm{C}\right) . \mathrm{IR}(\mathrm{KBr}):\left[\mathrm{cm}^{-1}\right]$ 3220, 3100, 1710, 1680, 1620; ${ }^{1} \mathrm{H}$ NMR (DMSO-d $): \delta 1.14(\mathrm{t}, J=7.5 \mathrm{~Hz}, 3 \mathrm{H}), 2.51$ (s, 3H), $3.11(\mathrm{~s}, 3 \mathrm{H}), 4.04$ (q, $J$ $=7.5 \mathrm{~Hz}, 2 \mathrm{H}), 5.19$ (d, $J=3.0 \mathrm{~Hz}, 1 \mathrm{H}), 7.21-7.39(\mathrm{~m}, 5 \mathrm{H}), 7.99$ (br s, $1 \mathrm{H})$.

Ethyl 1,6-dimethyl-4-(4-nitrophenyl)-2-oxo-1,2,3,4-tetrahydropyrimidine-5-

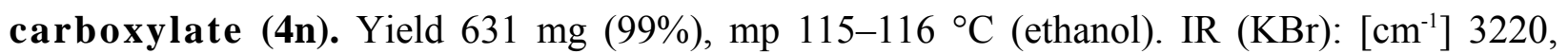
3090, $1710 \mathrm{sh}, 1700,1630 ;{ }^{1} \mathrm{H}$ NMR $\left(\mathrm{CDCl}_{3}\right): \delta 1.19(\mathrm{t}, J=7.5 \mathrm{~Hz}, 3 \mathrm{H}), 2.51(\mathrm{~s}, 1 \mathrm{H}), 3.21(\mathrm{~s}$, $1 \mathrm{H}), 4.10$ (q, $J=7.5 \mathrm{~Hz}, 2 \mathrm{H}), 5.48$ (d, J = 3.0 Hz, 1H), 6.54 (brs, 1H), 7.42 (d, J = $10 \mathrm{~Hz}, 2 \mathrm{H})$, $8.11(\mathrm{~d}, J=10.0 \mathrm{~Hz}, 2 \mathrm{H})$; Anal. calcd. for $\mathrm{C}_{15} \mathrm{H}_{17} \mathrm{~N}_{3} \mathrm{O}_{5}(319.32$ ): C, 56.42; H, 5.37; N 13.16. Found: C, 56.24; H, 5.24; N, 13.08 .

Ethyl 1,6-dimethyl-4-(3-nitrophenyl)-2-oxo-1,2,3,4-tetrahydropyrimidine-5carboxylate (4o). Yield $549 \mathrm{mg}(86 \%), \mathrm{mp} 126-127{ }^{\circ} \mathrm{C}$ (ethanol). IR (KBr): [cm $\left.{ }^{-1}\right] 3230$, 3090, $1700 \mathrm{sh}, 1680,1620 ;{ }^{1} \mathrm{H}$ NMR $\left(\mathrm{CDCl}_{3}\right): \delta 1.13$ (t, 3H, $\left.J=7.5 \mathrm{~Hz}\right), 2.50$ (s, 3H, C6-Me), 3.19 (s, 3H), 4.03 (q, J = 7.5 Hz, 2H), 5.44 (br s, 1H), 6.91 (br s, 1H), 7.30-7.60 (m, 2H), 7.968.18 (m, 2H); Anal. calcd. for $\mathrm{C}_{15} \mathrm{H}_{17} \mathrm{~N}_{3} \mathrm{O}_{5}$ (319.32): C, 56.42; H, 5.37; N, 13.16. Found: C, 56.07; H, 5.44; N, 13.12 .

Ethyl 1-benzyl-6-methyl-2-oxo-4-phenyl-1,2,3,4-tetrahydropyrimidine-5-carbo-xylate (4p). Yield $637 \mathrm{mg}$ (91\%), mp $157{ }^{\circ} \mathrm{C}$ (ethanol). IR (KBr): $\left[\mathrm{cm}^{-1}\right]$ 3210, 3100, 1710, 1690, 1620; ${ }^{1} \mathrm{H}$ NMR (DMSO-d6): $\delta 1.11(\mathrm{t}, J=7.5 \mathrm{~Hz}, 3 \mathrm{H}), 2.39$ (s, 3H), 4.03 (q, $J=7.5 \mathrm{~Hz}, 2 \mathrm{H}), 4.88,5.11$ (2 $\mathrm{d}, J=17.5 \mathrm{~Hz}, 2 \mathrm{H}), 5.25(\mathrm{~d}, J=3.0 \mathrm{~Hz}, 1 \mathrm{H}), 7.05-7.39(\mathrm{~m}, 5 \mathrm{H}), 8.13(\mathrm{~d}, J=3.0 \mathrm{~Hz}, 1 \mathrm{H})$; Anal. calcd. for $\mathrm{C}_{21} \mathrm{H}_{22} \mathrm{~N}_{2} \mathrm{O}_{3}(350.42)$ : C, 71.98; H, 6.33; N, 7.99. Found: C, 72.30; H, 6.52; N, 7.95.

Methyl 6-methyl-4-(2-methylphenyl)-2-oxo-1,2,3,4-tetrahydropyrimidine-5-carbo-xylate (4q). Yield $447 \mathrm{mg}$ (86\%), mp 240-242 ${ }^{\circ} \mathrm{C}$ (ethanol) (lit. $\left.{ }^{31} \mathrm{mp} \mathrm{240-242}{ }^{\circ} \mathrm{C}\right) . \mathrm{IR}(\mathrm{KBr}):\left[\mathrm{cm}^{-1}\right]$ 3230, 3100, 1695, $1665 \mathrm{~cm}^{-1} ;{ }^{1} \mathrm{H}$ NMR (DMSO-d $)$ ): $\delta 2.31$ (s, 3H), $2.43(\mathrm{~s}, 3 \mathrm{H}), 3.48(\mathrm{~s}, 3 \mathrm{H})$, 5.41 (d, $J=4.0 \mathrm{~Hz}, 1 \mathrm{H}), 7.11-7.22$ (m, 4H), 7.62 (br s, 1H), 9.19 (br s, 1H).

Methyl 6-methyl-4-(4-nitrophenyl)-2-oxo-1,2,3,4-tetrahydropyrimidine-5-carboxylate (4r). Yield $489 \mathrm{mg}(84 \%)$, mp 246-248 ${ }^{\circ} \mathrm{C}$ (acetic acid/ethanol) (lit. $\left.{ }^{13 \mathrm{c}} 235-237{ }^{\circ} \mathrm{C}\right) . \mathrm{IR}(\mathrm{KBr}):\left[\mathrm{cm}^{-1}\right.$ ] 3360, 3240, 3120, 1720, 1695, 1630; ${ }^{1} \mathrm{H}$ NMR (DMSO-d $)$ ): $\delta 2.29$ (s, 3H), 3.56 (s, 3H), 5.30 (br s, $1 \mathrm{H}), 7.53$ (d, $J=8.5 \mathrm{~Hz}, 2 \mathrm{H}), 7.92$ (br s, $1 \mathrm{H}), 8.23$ (d, $J=8.5 \mathrm{~Hz}, 2 \mathrm{H}), 9.40$ (br s, $1 \mathrm{H})$.

Isopropyl 6-methyl-2-oxo-4-phenyl-1,2,3,4-tetrahydropyrimidine-5-carboxylate (4s). Yield $485 \mathrm{mg}$ (84\%), mp $197^{\circ} \mathrm{C}$ (ethanol). IR (KBr): [ $\left.\mathrm{cm}^{-1}\right]$ 3240, 3120, 2980, 1720, 1700; ${ }^{1} \mathrm{H}$ NMR (DMSO-d $): \delta 1.00,1.19(2 \mathrm{~d}, J=7.5 \mathrm{~Hz}, 6 \mathrm{H}), 2.28(\mathrm{~s}, 1 \mathrm{H}), 4.82(\mathrm{~m}, 1 \mathrm{H}), 5.13(\mathrm{~d}, J=3.0 \mathrm{~Hz}$, $1 \mathrm{H}), 7.20-7.31(\mathrm{~m}, 5 \mathrm{H}), 7.71$ (br s, $1 \mathrm{H}), 9.17$ (br s, $1 \mathrm{H})$; Anal. calcd. for $\mathrm{C}_{15} \mathrm{H}_{18} \mathrm{~N}_{2} \mathrm{O}_{3}(274.32$ ): C, 65.68; H, 6.61; N, 10.21. Found: C, 65.39; H, 6.39; N, 9.84.

Isopropyl 6-methyl-4-(3-nitrophenyl)-2-oxo-1,2,3,4-tetrahydropyrimidine-5carbo-xylate (4t). Yield $587 \mathrm{mg}(88 \%)$, mp 205-208 ${ }^{\circ} \mathrm{C}$ (ethanol) (lit. ${ }^{22} 205-208{ }^{\circ} \mathrm{C}$ ). IR (KBr): $\left[\mathrm{cm}^{-1}\right]$ 3240, 3110, 1715sh, 1705, 1650; ${ }^{1} \mathrm{H}$ NMR (DMSO- $\left.d_{6}\right): \delta 1.00,1.18(2 \mathrm{~d}, J=6.0$ Hz each, 6H), $2.28(\mathrm{~s}, 3 \mathrm{H}), 4.84(\mathrm{~m}, 1 \mathrm{H}), 5.31(\mathrm{~d}, J=3.0 \mathrm{~Hz}, 1 \mathrm{H}), 7.65-7.77(\mathrm{~m}, 2 \mathrm{H}), 7.91$ (br s, 1H), 8.09-8.19 (m, 2H), 9.38 (br s, 1H).

tert.-Butyl 6-methyl-2-oxo-4-phenyl-1,2,3,4-tetrahydropyrimidine-5-carboxylate (4u). Yield $466 \mathrm{mg}$ (77\%), mp 216-217 ${ }^{\circ} \mathrm{C}$ (ethanol) (lit. $\left.{ }^{27} 209{ }^{\circ} \mathrm{C}\right) . \mathrm{IR}(\mathrm{KBr}):\left[\mathrm{cm}^{-1}\right]$ 3240, 3090, 1715sh, 
1705, 1680, 1650; ${ }^{1} \mathrm{H}$ NMR (DMSO-d $): \delta 1.30(\mathrm{~s}, 9 \mathrm{H}), 2.24(\mathrm{~s}, 3 \mathrm{H}), 5.12(\mathrm{~d}, J=3.0 \mathrm{~Hz}, 1 \mathrm{H})$, 7.20-7.39 (m, 5H), 7.69 (br s, 1H), 9.09 (br s, 1H).

Benzyl 1,6-dimethyl-2-oxo-4-phenyl-1,2,3,4-tetrahydropyrimidine-5-carboxylate (4v). Yield $624 \mathrm{mg}(93 \%), \mathrm{mp} 152{ }^{\circ} \mathrm{C}$ (ethanol) (lit. $\left.{ }^{1} 152{ }^{\circ} \mathrm{C}\right) . \mathrm{IR}(\mathrm{KBr}):\left[\mathrm{cm}^{-1}\right]$ 3240, 3110, 1705, 1680, 1625; ${ }^{1} \mathrm{H}$ NMR $\left(\mathrm{CDCl}_{3}\right): \delta 2.57(\mathrm{~s}, 3 \mathrm{H}), 3.14(\mathrm{~s}, 3 \mathrm{H}), 4.96-5.16(\mathrm{~m}, 2 \mathrm{H}), 5.74(\mathrm{~d}, J=3.5 \mathrm{~Hz}$, $1 \mathrm{H}), 7.45-7.60(\mathrm{~m}, 10 \mathrm{H}), 8.0(\mathrm{~d}, J=3.5 \mathrm{~Hz}, 1 \mathrm{H})$.

Benzyl 4-(2,3-dichlorophenyl)-1,6-dimethyl-2-oxo-1,2,3,4-tetrahydropyrimidine-5carboxylate (4w). Yield $725 \mathrm{mg}(93 \%), \mathrm{mp} 128-131^{\circ} \mathrm{C}$ (ethanol). IR (KBr): [cm $\left.{ }^{-1}\right]$ 3240, 3110, 1700, 1680, 1624; ${ }^{1} \mathrm{H} \mathrm{NMR}\left(\mathrm{CDCl}_{3}\right): \delta 2.57$ (s, 3H), $3.14(\mathrm{~s}, 3 \mathrm{H}), 4.96-5.16(\mathrm{~m}, 2 \mathrm{H}), 5.74(\mathrm{~d}, J$ $=3.5 \mathrm{~Hz}, 1 \mathrm{H}), 6.90-7,60(\mathrm{~m}, 8 \mathrm{H}), 8.1(\mathrm{~d}, J=3.5 \mathrm{~Hz}, 1 \mathrm{H})$. Anal. calcd.. for $\mathrm{C}_{20} \mathrm{H}_{18} \mathrm{Cl}_{2} \mathrm{~N}_{2} \mathrm{O}_{3}$ : C, 59.27; H, 4.48; N, 6.91. Found: C, 59.38; H, 4.41; N, 6.87.

Allyl 1,6-dimethyl 4-(3-nitrophenyl)-2-oxo-1,2,3,4-tetrahydropyrimidine-5-carboxylate (4x). Yield $562 \mathrm{mg}(85 \%), \mathrm{mp} 125-126^{\circ} \mathrm{C}$ (ethanol). IR (KBr): $\left[\mathrm{cm}^{-1}\right]$ 3240, 3110, 1700, 1680, 1630; ${ }^{1} \mathrm{H}$ NMR $\left(\mathrm{CDCl}_{3}\right): \delta 2.51(\mathrm{~s}, 3 \mathrm{H}), 3.20(\mathrm{~s}, 3 \mathrm{H}), 4.52(\mathrm{~m}, 2 \mathrm{H}), 5.10-5.20(\mathrm{~m}, 2 \mathrm{H}), 5.45(\mathrm{~d}, J=$ $3.5,1 \mathrm{H}), 5.70-5.90(\mathrm{~m}, 1 \mathrm{H}), 6.82(\mathrm{~d}, J=3.5,1 \mathrm{H}), 7.30-7.60(\mathrm{~m}, 2 \mathrm{H}), 8.00-8.10(\mathrm{~m}, 2 \mathrm{H})$. Anal. calcd.. for $\mathrm{C}_{16} \mathrm{H}_{17} \mathrm{~N}_{3} \mathrm{O}_{5}$ : C, 58.00; H, 5.17; N, 12.68. Found: 58.30; H, 5.11; N, 12.50 .

Ethyl 6-methyl-2-oxo-4-(phenylethinyl)-1,2,3,4-tetrahydropyrimidine-5-carboxylate (4y). Yield $600 \mathrm{mg}(92 \%), \mathrm{mp} 226^{\circ} \mathrm{C}$ (ethanol). IR (KBr): [ $\left.\mathrm{cm}^{-1}\right]$ 3220, 3100, $1730 \mathrm{sh}, 1710,1660 ;{ }^{1} \mathrm{H}$ NMR (DMSO- $\left.d_{6}\right): \delta 1.26(\mathrm{t}, J=7.5 \mathrm{~Hz}, 3 \mathrm{H}), 2.28(\mathrm{~s}, 1 \mathrm{H}), 4.15(\mathrm{~m}, J=7.5 \mathrm{~Hz}, 2 \mathrm{H}), 5.13(\mathrm{~d}, J=$ $3.0 \mathrm{~Hz}, 1 \mathrm{H}), 7.40$ (s, 5H), 7.80 (br s, 1H), 9.40 (brs, 1H); ${ }^{13} \mathrm{C}$ NMR (DMSO-d6): $\delta$ 14.26, 17.67, 42.28, 59.45, 80.82, 90.21, 96.77, 121.98, 128.59, 128.67, 131.30, 149.24, 152.27, 164.64; Anal. calcd. for $\mathrm{C}_{16} \mathrm{H}_{16} \mathrm{~N}_{2} \mathrm{O}_{3}$ : C, 67.59; H, 5.67; N, 9.85. Found: C, 67.41; H, 5.51; N, 9.67.

Ethyl 4,6-dimethyl-2-oxo-1,2,3,4-tetrahydropyrimidine-5-carboxylate (4z). Yield $210 \mathrm{mg}$ (53\%), mp 193-195 ${ }^{\circ} \mathrm{C}$ (ethanol) (lit. ${ }^{23} 189-190{ }^{\circ} \mathrm{C}$ ). IR (KBr): $\left[\mathrm{cm}^{-1}\right] 3260,3100,1700,1650 ;{ }^{1} \mathrm{H}$ NMR (DMSO-d $\left.)_{6}\right): \delta 1.11((\mathrm{~d}, J=5.5 \mathrm{~Hz}, 3 \mathrm{H}), 1.20(\mathrm{t}, \mathrm{J}=7.5 \mathrm{~Hz}, 3 \mathrm{H}), 2.19(\mathrm{~s}, 3 \mathrm{H}), 4.00-4.22$ (m, 3H), 7.20 (br s, 1H), 8.99 (br s, 1H).

Ethyl (E)-2-oxo-4-phenyl-6-styryl-1,2,3,4-tetrahydropyrimidine-5-carboxylate (18). A mixture of DHPM 4a (260 mg, $1 \mathrm{mmol})$, benzaldehyde (212 mg, $2 \mathrm{mmol}$ ) and $p$-toluenesulfonic acid (ca. $10 \mathrm{mg}$ ) in xylene $(10 \mathrm{~mL})$ was heated under reflux for $48 \mathrm{~h}$. After all starting material was consumed (TLC), the solution was cooled to ambient temperature. The precipitated crude product was purified by recrystallization from ethanol to give 18 (201 mg, 58\%) , mp 245-247 ${ }^{\circ} \mathrm{C}$. IR (KBr): $\left[\mathrm{cm}^{-1}\right]$ 3230, 3190, $1710 \mathrm{sh}, 1700,1640 ;{ }^{1} \mathrm{H} \mathrm{NMR}\left(\mathrm{CDCl}_{3}\right): \delta 1.21(\mathrm{t}, \mathrm{J}=7.5 \mathrm{~Hz}$, $3 \mathrm{H}), 4.14(\mathrm{q}, J=7.5 \mathrm{~Hz}, 2 \mathrm{H}), 5.50(\mathrm{~d}, J=3.0 \mathrm{~Hz}, 1 \mathrm{H}), 5.83$ (br s, 1H), 7.12 (d, $J=16.0 \mathrm{~Hz}$, 1H), 7.23-7.58 (m, 10H), $7.82(\mathrm{~s}, 1 \mathrm{H}), 8.09$ (d, J = 16.0 Hz, 1H); ${ }^{13} \mathrm{C}$ NMR (DMSO-d $)$ ) d 14.0, 54.00, 59.80, 102.04, 119.60, 126.28, 127.142, 127.46, 128.51, 128.98, 129.09, 134.79, 135.99, 144.32, 144.59, 152.54, 165.16; Anal. calcd. for $\mathrm{C}_{21} \mathrm{H}_{20} \mathrm{~N}_{2} \mathrm{O}_{3}: \mathrm{C}, 72.40 ; \mathrm{H}, 5.79 ; \mathrm{N}, 8.04$. Found: C, 72.38; H, 5.89; N, 8.02. 


\section{Acknowledgements}

This work was supported by the Austrian Academy of Sciences (APART 319) and the Austrian Science Fund (FWF, Project P-11994-CHE).

\section{References}

1. Synthesis and Reactions of Biginelli compounds, 24. Part 23: Schnell, B.; Krenn, W.; Faber, K.; Kappe, C. O. J. Chem. Soc., Perkin Trans. 1 2000, 4382.

2. Biginelli, P. Gazz. Chim. Ital. 1893, 23, 360.

3. Review: Kappe, C. O. Tetrahedron 1993, 49, 6937.

4. Review: Kappe, C. O. Acc. Chem. Res. 2000, 33, 879.

5. Review. Kappe, C. O. Eur. J. Med. Chem. 2000, 35, 1043.

6. Atwal, K. S.; Swanson, B. N.; Unger, S. E.; Floyd, D. M.; Moreland, S.; Hedberg, A.; O'Reilly, B. C. J. Med. Chem. 1991, 34, 806.

7. Rovnyak, G. C.; Atwal, K. S.; Hedberg, A.; Kimball, S. D.; Moreland, S.; Gougoutas, J. Z.; O'Reilly, B. C.; Schwartz, J.; Malley, M. F. J. Med. Chem. 1992, 35, 3254.

8. Grover, G. J.; Dzwonczyk, S.; McMullen, D. M.; Normandin, D. E.; Parham, C. S.; Sleph, P. G.; Moreland, S. J. Cardiovasc. Pharm. 1995, 26, 289.

9. (a) Nagarathnam, D.; Miao, S. W.; Lagu, B.; Chiu, G.; Fang, J.; Dhar, T. G. M.; Zhang, J.; Tyagarajan, S.; Marzabadi, M. R.; Zhang, F. Q.; Wong, W. C.; Sun, W. Y.; Tian, D.; Wetzel, J. M.; Forray, C.; Chang, R. S. L.; Broten, T. P.; Ransom, R. W.; Schorn, T. W.; Chen, T. B.; O'Malley, S.; Kling, P.; Schneck, K.; Benedesky, R.; Harrell, C. M.; Vyas, K. P.; Gluchowski, C. J. Med. Chem. 1999, 42, 4764, and subsequent papers in this issue. (b) Barrow, J. C.; Nantermet, P. G.; Selnick, H. G.; Glass, K. L.; Rittle, K. E.; Gilbert, K. F.; Steele, T. G.; Homnick, C. F.; Freidinger, R. M.; Ransom, R. W.; Kling, P.; Reiss, D.; Broten, T. P.; Schorn, T. W.; Chang, R. S. L.; O'Malley, S. S.; Olah, T. V.; Ellis, J. D.; Barrish, A; Kassahun, K.; Leppert, P.; Nagarathnam, D.; Forray, C. J. Med. Chem. 2000, 43 2703.

10. (a) Mayer, T. U.; Kapoor, T. M.; Haggarty, S. J.; King, R. W.; Schreiber, S. L.; Mitchison, T. J. Science 1999, 286, 971.(b) Haggarty, S. J.; Mayer, T. U.; Miyamoto, D. T.; Fathi, R.; King, R. W.; Mitchison, T. J.; Schreiber, S. L. Chem. Biol. 2000, 7, 275.

11. Review: Heys, L.; Moore, C. G.; Murphy, P. J. Chem. Soc. Rev. 2000, $29,57$.

12. Patil, A. D.; Kumar, N. V.; Kokke, W. C.; Bean, M. F.; Freyer, A. J.; De Brosse, C.; Mai, S.; Truneh, A.; Faulkner, D. J.; Carte, B.; Breen, A. L.; Hertzberg, R. P.; Johnson, R. K.; Westley, J. W.; Potts, B. C. M. J. Org. Chem. 1995, 60, 1182.

13. (a) Lu, J.; Ma, H. Synlett 2000, 63. (b) Ma, Y.; Qian, C.; Wang, L.; Yang, M. J. Org. Chem. 2000, 65, 3864. (c) Hu, E. H.; Sidler, D. R.; Dolling, U.-H. J. Org. Chem. 1998, 63, 3454. (d) Shutalev, A. D.; Sivova, N. V. Khim. Geterotsikl. Soedin. 1998, 979. (e) Dandia, A.; Saha, M.; Taneja, H. J. Fluorine Chem. 1998, 90, 17. (f) Gupta, R.; Gupta, A. K.; Paul, S.; 
Kachroo, P. L. Ind. J. Chem. 1995, 34B, 151.

14. (a) O'Reilly, B. C.; Atwal, K. S. Heterocycles 1987, 26, 1185. (b) Atwal, K. S.; O' Reilley, B. C.; Gougoutas, J. Z.; Malley, M. F. Heterocycles 1987, 26, 1189. (c) Shutalev, A. D.; Kuksa, V. A. Khim. Geterotsikl. Soedin. 1997, 105. (d) Shutalev, A. D.; Kishko, E. A.; Sivova, N.; Kuznetsov, A. Y. Molecules 1998, 3, 100.

15. (a) Wipf, P.; Cunningham, A. Tetrahedron Lett. 1995, 36, 7819. (b) Kappe, C. O. Bioorg. Med. Chem. Lett. 2000, 10, 49. (c) Studer, A.; Hadida, S.; Ferrito, R.; Kim, S.-Y.; Jeger, P.; Wipf, P.; Curran, D. P. Science 1997, 275, 823. (d) Studer, A.; Jeger, P.; Wipf, P.; Curran, D. P. J. Org. Chem. 1997, 62, 2917. (e) Lewandowski, K.; Murer, P.; Svec, F.; Fréchet, J. M. J. Chem. Commun. 1998, 2237.

16. Kappe, C. O. J. Org. Chem. 1997, 62, 7201.

17. Petersen, H. Synthesis 1973, 243.

18. (a) Overman, L. E.; Ricca, D. J. Comprehensive Organic Synthesis; Trost, B. M.; Fleming, I.; Eds; Pergamon Press: Oxford, 1991; Vol. 2, p 1007. (b) Hiemstra, H.; Speckamp, W. N. Comprehensive Organic Synthesis; Trost, B. M.; Fleming, I.; Ed.; Pergamon Press: Oxford 1991; Vol. 2, p 1047, and references cited therein.

19. Dixon, L. A. In Encyclopedia of Reagents for Organic Synthesis, Paquette, L., Ed.; Wiley: Chichester, 1995; Vol. 6, p 4166.

20. Marson, C. M.; Grabowska, U.; Fallah, A.; Walsgrove, T.; Eggleston, D. S.; Baures, P. W. J. Org. Chem. 1994, 59, 291.

21. For a preliminary report, see: Kappe, C. O.; Falsone, S. F. Synlett 1998, 718.

22. Kappe, C. O.; Kumar, D.; Varma, R. S. Synthesis 1999, 1799.

23. Folkers, K.; Harwood, H. J.; Johnson, T. B. J. Am. Chem. Soc. 1932, 54, 3751.

24. (a) Shutalev, A. D.; Sivova, N. V. Khim. Geterotsikl. Soedin. 1998, 979. (b) Stadler, A.; Kappe, C. O. J. Chem. Soc., Perkin Trans. 2 2000, 1363.

25. Stadler, A.; Kappe, C. O. J. Chem. Soc., Perkin Trans. 2 2000, 1363.

26. Khromov-Borisov, M. V.; Savchenko, A. M. Zh. Obshch. Khim. 1952, 22, 1680.

27. Zigeuner, G.; Knopp, C.; Blaschke, H. Monatsh. Chem. 1976, 107, 587.

28. Kappe, C. O.; Wagner, U. G. Heterocycles 1989, 29, 761.

29. McKinstry, D. W.; Reading, E. H.; J. Franklin Inst. 1944, 237, 203.

30. Folkers, K.; Johnson, T. B. J. Am. Chem. Soc. 1933, 55, 2886.

31. Kappe, C. O.; Fabian, W. M. F.; Semones, M. A. Tetrahedron 1997, 53, 2803. 